


\title{
Modelo de ruteo capacitado con flota heterogénea para la distribución de pedidos: caso aplicado a una empresa ladrillera de la ciudad de Sincelejo, Sucre
}

\author{
Leonardo Arcos Domínguez ${ }^{1}$, Rohonal Rodríguez Salgado², \\ William Torres Meza ${ }^{3}$, Ronaldo Vecino Vargas ${ }^{4}$
}

\section{Resumen}

Dentro de todos los procesos que conforman la cadena de suministro de una actividad productiva, el factor transporte es considerado uno de los más importantes para el adecuado funcionamiento de esta, puesto que es el encargado de la movilización de los recursos necesarios para que se lleven a cabo todos los procesos de la cadena en general. Cabe mencionar que este factor representa uno de los costos de mayor relevancia dentro del proceso logístico, por lo cual es necesario la aplicación de herramientas o metodologías que permitan reducirlos, entre las que se encuentra el sistema de ruteo estratégico. Este último, dentro de su operatividad, busca minimizar los costos de transporte, teniendo en cuenta la mejor ruta para la distribución de los productos. La presente investigación tiene como finalidad diseñar un modelo de ruteo estratégico para la distribución de ladrillos en la empresa Cerámicas El Cinco, donde se detectaron altos costos de transporte ocasionados por la falta de planeación con respecto al reparto de pedidos. Para este diseño fue necesario la utilización de la herramienta GAMS, la cual permitió codificar el modelo y encontrar

1 Ingeniero Industrial. Corporación Universitaria del Caribe-CECAR. Correo: leonardo.arcos@cecar.edu.co; Orcid: https://orcid.org/0000-0001-8464-0379

2 Ingeniero Industrial. Corporación Universitaria del Caribe-CECAR. Correo: rohonal.rodriguez@cecar.edu.co; Orcid: https://orcid.org/0000-0003-4273-3133

3 Ingeniero Industrial. Corporación Universitaria del Caribe-CECAR.william. torres@cecar.edu.co; Orcid: https://orcid.org/0000-0002-2785-0174

4 Ingeniero Industrial. Corporación Universitaria del Caribe-CECAR. Correo: ronaldo.vecino@cecar.edu.co; Orcid: https://orcid.org/0000-0003-2963-5432 
la mejor solución, obteniéndose una disminución del 11,69\% en los costos de transporte.

Palabras clave: logística, distribución, cadena de suministro, transporte, problema de ruteo de vehículos.

\section{Capacitated routing model with heterogeneous fleet for order distribution: case applied to a brickyard company in the city of Sincelejo, Sucre.}

\section{Abstract}

Within all the processes that make up the supply chain of a productive activity, the transport factor is considered one of the most important for the proper functioning of this, since it is responsible for the mobilization of the necessary resources to be carried All processes of the chain in general, it should be mentioned that this represents one of the most relevant costs in the logistics process, which is why it is necessary to apply tools or methodologies to reduce them; within which is, the strategic routing system, which within its operation, seek to minimize transport costs, taking into account the best route for the distribution of products. Now, the purpose of this research is to design a strategic routing model for the distribution of bricks in the company CERAMICAS EL CINCO, where high transportation costs were detected due to the lack of planning regarding the distribution of orders. For this design it was necessary to use tools such as GAMS, which allowed coding the model and finding the best solution, obtaining in this a decrease of $11.69 \%$ in transportation costs.

Keywords: logistics, distribution, supply chain, transportation, vehicle routing problem. 


\section{Introducción}

En la actualidad, el entorno laboral se ha tornado demasiado exigente, lo cual ha llevado a que las empresas, que quieran sobrevivir y posicionarse, diseñen e implementen estrategias y técnicas para aumentar su ventaja competitiva y, sobre todo, para detectar, corregir y prevenir las problemáticas que se puedan presentar en las tareas vinculadas a su cadena de suministro.

Una de las técnicas implementadas en las empresas para dar solución a las actividades vinculadas a su cadena de suministro es la gestión de cadena de suministro (SCM), la cual, si se lleva a cabo de manera adecuada, ayuda a las organizaciones a la transformación de su cadena en un proceso óptimo y eficiente que satisfaga plenamente las necesidades y expectativas del cliente final; teniendo como base que la eficiencia de todos sus eslabones integrados es mucho más importante que la de cada cual por separado.

La caracterización de la cadena de suministro llevada a cabo en este escrito se basa en la identificación de todas las tareas que se encuentran vinculadas al proceso y al seguimiento que se les debe realizar para poder controlarlas. Esto permite a la empresa tener clara la relación existente con los demás procesos anexos, facilitando a todos los empleados de la organización conocer de modo sencillo cómo opera esta y de qué manera se gestionan sus procesos.

La cadena de suministro es un proceso que abarca el flujo continuo de materias primas, recursos necesarios e información requerida mediante diversas áreas, entre las partes que se encuentran involucradas en la cadena, permitiendo suministrar con mayor facilidad las operaciones que se puedan llevar a cabo a futuro en pro del aumento de la ventaja competitiva (Gutiérrez, Fuquen, \& Abril , 2010). Cabe resaltar que la esquematización, análisis y correcta gestión de la cadena de suministro ayuda a identificar, corregir y prevenir problemas, con mayor facilidad, de los procesos tanto internos como externos de una organización.

Actualmente, la logística ha ido progresando de tal manera que es catalogada como una de las técnicas o herramientas más importantes para el aumento de la ventaja competitiva de una empresa, enfocándose con mayor ahínco en los aspectos que comprenden el transporte, resaltando que es 
uno de los elementos más importantes dentro de la cadena de suministro. Este tiene como esencia brindar, en términos de seguridad, regularidad, oportunidad, entregas a tiempo y costos, un servicio de calidad, ya sea desde que se realiza el aprovisionamiento de materias primas hasta que se entrega el producto al cliente final (Chopra \& Meindl, 2016); este proceso es uno de los que mayores costos logísticos genera (Ballou, 2004).

Ahora bien, una de las alternativas para contrarrestar la problemática mencionada es lo que respecta a la aplicación de un sistema de ruteo estratégico, el cual busca minimizar los costos de transporte mediante la elección de la ruta más conveniente, es decir, aquella que cumpla con todas las especificaciones y que contribuya a la mejora del problema (Joshi $\&$ Kaur, 2015 ; Daza, Montoya, \& Narducci, 2013).

Por tal razón, el presente artículo tiene como finalidad presentar un modelo de ruteo capacitado con flota heterogénea para el reparto de ladrillos en la empresa Cerámicas El Cinco.

\section{Materiales y métodos}

La metodología empleada en la presente investigación es de carácter descriptivo y abarca distintas fases:

Fase 1. Caracterización de la cadena de suministro: en esta fase se analiza cómo se encuentra la cadena de suministro del ladrillo a nivel mundial, nacional y regional; luego se estudia detalladamente la cadena de suministro escogida para conocer todos sus procesos internos y la manera como se lleva a cabo el desarrollo de las actividades que la involucran.

Fase 2. Recolección de datos: luego de analizar las condiciones presentes en la cadena de suministro, y con la convicción de poder desarrollar el diseño de un modelo que le brinde una buena solución, se realiza el proceso de recolección de información en cuanto a variables y parámetros, el cual tiene como fin conocer y obtener los datos necesarios para la ejecución del modelo.

Fase 3. Formulación del modelo matemático: dada la recolección de datos, se procede al desarrollo del modelo de ruteo, el cual tiene como fin minimizar los altos costos de transporte que genera la inadecuada distribución de los productos. 
Fase 4. Evaluación del modelo con datos reales y análisis de resultados: es aquí donde se procede a analizar toda la información arrojada por el modelo matemático una vez codificado y, con base en los criterios definidos, establecer las posibles conclusiones.Diagnóstico actual de la cadena de suministro del ladrillo

Tras un análisis detallado de la cadena de suministro del ladrillo a nivel mundial, se pudo determinar que esta se encuentra constituida por 5 eslabones (proveedores, acopiadores, productores, distribuidores y clientes), asimismo, cabe mencionar que a nivel nacional esta presenta una estructura similar, la cual se encuentra conformada por los 5 eslabones ya mencionados, como se puede apreciar en la figura 1. Sin embargo, es necesario resaltar que a nivel departamental la cadena de suministro presenta cierta diferencia en cuanto a su estructura, puesto que esta se encuentra conformada básicamente por 4 eslabones (proveedores, acopioproducción, comercialización y clientes), como se representa en la figura 2. Esta diferencia se debe a que las empresas transformadoras o productoras cuentan con zonas de almacenamiento propias para acopiar los materiales suministrados y, a su vez, los productos finales.

Para ser más detallados, las empresas a nivel mundial o nacional presentan una alta demanda de ladrillos y, por ende, necesitan de empresas acopiadoras para almacenar los productos mientras que a nivel departamental la demanda, en comparación con las demás, es mucho menor y, por ello, las empresas tienen la capacidad para almacenar sus productos en sus centros o zonas de acopio. Las cifras en cuanto a producción de ladrillos a nivel nacional se pueden observar en la figura 3. 
Modelo de ruteo capacitado con flota heterogénea para la distribución de pedidos: caso aplicado a una empresa ladrillera de la ciudad de Sincelejo, Sucre

Figura 1

Cadena de suministro a nivel mundial y nacional.

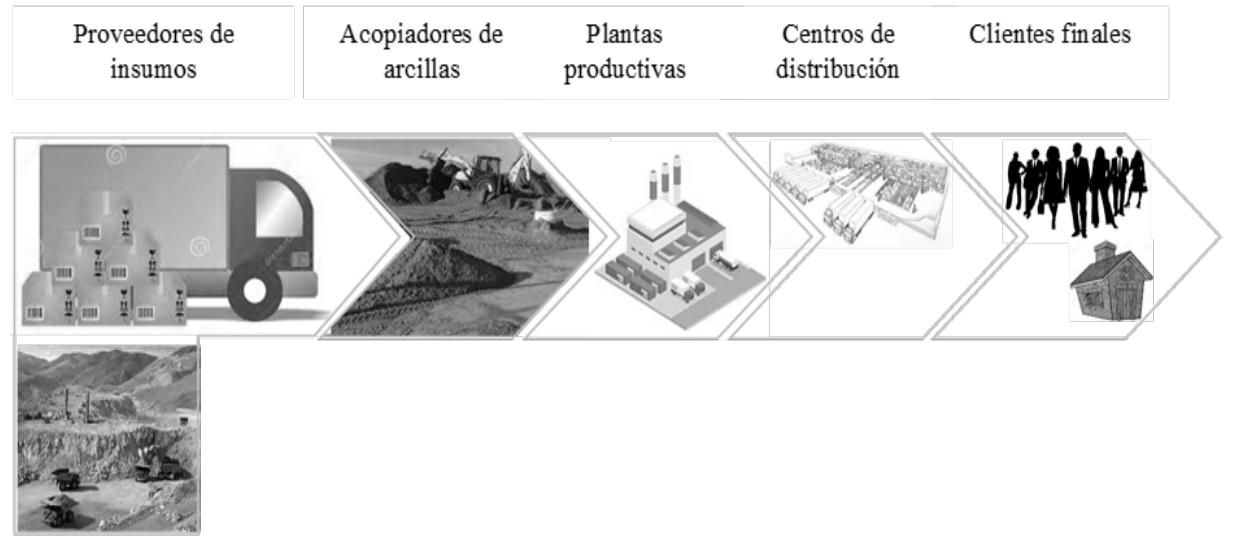

Nota. Fuente: construcción propia.

Figura 2

Cadena de suministro a nivel departamental.
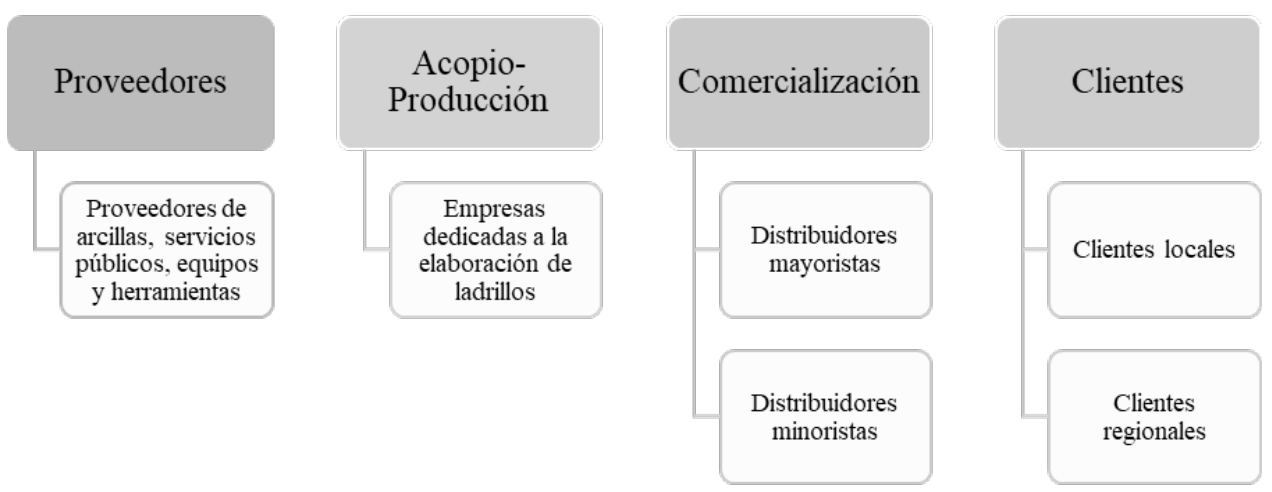

Nota. Fuente: construcción propia. 
Figura 3

Producción por unidad de ladrillos a nivel nacional.

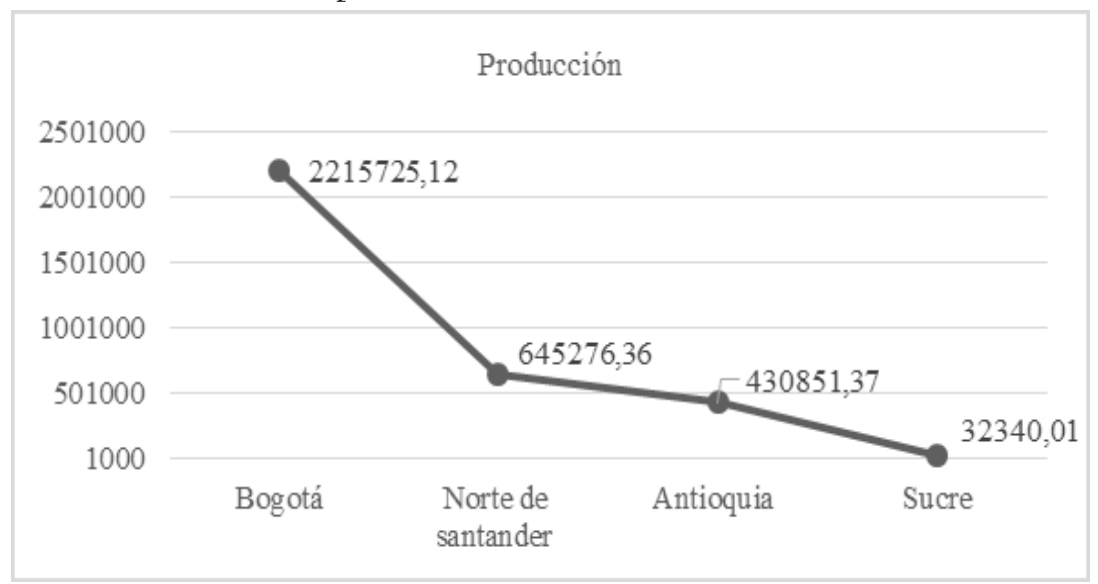

Nota. Fuente: construcción propia.

Luego de un estudio de la cadena de suministro en cuestión, se pudo identificar que la problemática que mayor impacto genera son los altos costos de transporte, los cuales son generados debido a la mala planeación en la distribución de los productos. Por tal razón, cabe mencionar que los costos de transporte por día que actualmente Cerámicas El Cinco posee se estiman en alrededor de los \$3.550.000.Recolección de información en la empresa

Para la compilación del modelo de ruteo propuesto fue indispensable la recopilación de información en cuanto a clientes, demandas, costos de transporte, cantidad de camiones, tipo de camiones, tipo de productos, entre otros, los cuales fueron obtenidos gracias a la colaboración de la empresa Cerámicas El Cinco. (Figuras 1, 2, 3, 4, 5, 6). 
Modelo de ruteo capacitado con flota heterogénea para la distribución de pedidos: caso aplicado a una empresa ladrillera de la ciudad de Sincelejo, Sucre

Tabla 1

Referencias de Productos.

\begin{tabular}{lc}
\hline \multicolumn{1}{c}{ Tipo } & Referencia \\
\hline Ladrillo para levante de muro $N^{\circ} 4$ & 1 \\
Ladrillo para levante de muro $N^{\circ} 5^{\circ}$ & 2 \\
Ladrillo para perforación vertical $N^{\circ} 4$ & 3 \\
Ladrillo para perforación vertical No 5 & 4 \\
Ladrillo Catalán $N^{\circ} 6$ & 5 \\
Ladrillo Catalán $N^{\circ} 9$ & 6 \\
Ladrillo Bloquelón & 7 \\
\hline
\end{tabular}

Nota. Fuente: construcción propia.

Tabla 2

Masa de Productos.

\begin{tabular}{cc}
\hline Ladrillo & Masa (ton) \\
\hline 1 & 0,0045 \\
2 & 0,0073 \\
3 & 0,0068 \\
4 & 0,0075 \\
5 & 0,002 \\
6 & 0,0031 \\
7 & 0,011 \\
\hline
\end{tabular}

Nota. Fuente: construcción propia.

Tabla 3

Capacidad de camiones.

\begin{tabular}{ccc}
\hline Tipo & Camión & Capacidad (ton) \\
\hline \multirow{2}{*}{$\mathrm{N}^{\circ} 1$} & 1 & 10 \\
& 2 & 10 \\
& 3 & 15 \\
$\mathrm{~N}^{\circ} 2$ & 4 & 15 \\
& 5 & 15 \\
\hline
\end{tabular}

Nota. Fuente: construcción propia. 
Tabla 4

Costo fijo por uso del vehículo (miles de pesos).

\begin{tabular}{ccc}
\hline Tipo & Camión & Costo \\
\hline \multirow{2}{*}{$\mathrm{N}^{\circ} 1$} & 1 & 85 \\
& 2 & 85 \\
& 3 & 125 \\
$\mathrm{~N}^{\circ} 2$ & 4 & 125 \\
& 5 & 125 \\
\hline
\end{tabular}

Nota. Fuente: construcción propia.

Tabla 5

Costo de viajar desde el destino hasta el cliente (miles de pesos).

\begin{tabular}{|c|c|c|c|c|c|c|c|c|}
\hline $\begin{array}{l}\text { Destino/ } \\
\text { Clientes }\end{array}$ & 1 & 2 & 3 & 4 & 5 & 6 & 7 & 8 \\
\hline 1 & 0 & 470 & 450 & 435 & 445 & 245 & 260 & 255 \\
\hline 2 & 410 & 0 & 40 & 65 & 55 & 690 & 705 & 700 \\
\hline 3 & 390 & 40 & 0 & 45 & 40 & 670 & 685 & 680 \\
\hline 4 & 365 & 65 & 45 & 0 & 45 & 655 & 670 & 665 \\
\hline 5 & 380 & 55 & 40 & 45 & 0 & 665 & 680 & 675 \\
\hline 6 & 180 & 690 & 670 & 655 & 665 & 0 & 40 & 35 \\
\hline 7 & 195 & 705 & 685 & 670 & 680 & 40 & 0 & 35 \\
\hline 8 & 185 & 700 & 680 & 665 & 675 & 35 & 35 & 0 \\
\hline
\end{tabular}

Tabla 6

Demanda de los productos por parte de los clientes (unidades).

\begin{tabular}{ccccccccc}
\hline Productos/clientes & $\mathbf{1}$ & $\mathbf{2}$ & $\mathbf{3}$ & $\mathbf{4}$ & $\mathbf{5}$ & $\mathbf{6}$ & $\mathbf{7}$ & $\mathbf{8}$ \\
\hline 1 & 0 & 730 & 810 & 0 & 820 & 0 & 900 & 750 \\
2 & 0 & 245 & 326 & 420 & 0 & 350 & 250 & 0 \\
3 & 0 & 0 & 0 & 0 & 480 & 200 & 0 & 150 \\
4 & 0 & 0 & 0 & 0 & 240 & 100 & 0 & 70 \\
5 & 0 & 0 & 0 & 320 & 0 & 0 & 0 & 0 \\
6 & 0 & 0 & 0 & 275 & 0 & 0 & 0 & 0 \\
7 & 0 & 0 & 0 & 300 & 0 & 350 & 0 & 150 \\
\hline
\end{tabular}

Nota. Fuente: construcción propia. 


\section{Modelo propuesto}

Modelo conceptual: Este problema de ruteo capacitado con flota heterogénea (CHVRP) puede ser formalizado de la siguiente manera: Sea $\mathrm{G}=(\mathrm{V}, \mathrm{E})$ un grafo no dirigido donde $\mathrm{V}=\{1, \ldots, \mathrm{m}+\mathrm{n}\}$ corresponde al conjunto de vértices y $\mathrm{E}$ al conjunto de arcos. Los vértices J corresponden a los clientes con demanda de cada producto $d_{j m}$ y los vértices I corresponden a los depósitos.

Un costo variable $C_{i j}$ es asociado a cada par de nodos $(\mathbf{i}, \mathbf{j}) \in \mathrm{E}$. Un conjunto de vehículos heterogéneos $\mathrm{K}$, cada uno con capacidad $q_{k}$ está disponible en el depósito i $\in \mathrm{I}$. Asimismo, cada vehículo $\mathrm{k} \in K$ cuando realiza una ruta genera un costo fijo $v_{k}$. El objetivo principal de CHVRP es minimizar los costos logísticos de transporte, incluyendo los costos fijos por uso de los vehículos, también los costos variables asociados a cada ruta establecida. Este CHVRP es mono objetivo, con restricciones y discreto.

Una solución factible para el CHVRP debe satisfacer las siguientes restricciones: i) cada ruta establecida debe iniciar y finalizar en el depósito, ii) cada cliente debe ser visitado estrictamente una sola vez por una sola ruta asignada a un vehículo $\mathrm{k} \in \mathrm{K}$, iii) el total de las demandas de los clientes visitados por el vehículo $\mathrm{k} \in \mathrm{K}$ no puede exceder su capacidad $q_{k}$, iv) el flujo entre depósitos no es admitido.

Modelo matemático: En esta ocasión se formula un modelo de programación entera mixta para el CHVRP que cuenta con cuatro conjuntos:

$i$ : Depósito

$j$ : Clientes

$k$ : Vehículos

$m$ : Productos

Por otra parte, el modelo cuenta con los siguientes parámetros:

$\mathrm{V}_{k}=$ costo fijo por utilización del vehículo $\mathrm{k}$

$\mathrm{c}_{i j}=$ costo de viajar desde el nodo i al nodo $\mathrm{j}$

$\mathrm{mp}_{m}=$ masa del producto $\mathrm{m}$

$\mathrm{q}_{k}=$ capacidad de carga del vehículo $\mathrm{k}$ 
$d_{j m}=$ demanda del cliente $\mathrm{j}$ del producto $\mathrm{m}$

$m g$ = número muy grande para acotar las cantidades a transportar

Esta formulación emplea las siguientes variables enteras y binarias:

$z u_{i j k m}=$ unidades del producto a transportar de un nodo a un nodo en un vehículo .

$X_{i j k}=$ toma valores de 1 si se utiliza el arco dea en la ruta asignada al vehículo $\mathrm{k} \in \mathrm{K}$.

Función objetivo

$$
\begin{aligned}
& \operatorname{Min} \mathrm{Z}=\sum_{\mathrm{i} \in \mathrm{V}} \sum_{\mathrm{j} \in \mathrm{V}} \sum_{\mathrm{k} \in \mathrm{K}} \mathrm{c}_{\mathrm{ij}} * \mathrm{x}_{\mathrm{ijk}}+\sum_{\mathrm{i} \in \mathrm{I}} \sum_{\mathrm{j} \in \mathrm{J}} \sum_{\mathrm{k} \in \mathrm{K}} \mathrm{v}_{\mathrm{k}} * \mathrm{x}_{\mathrm{ijk}} \\
& \sum_{\mathrm{k} \in \mathrm{K}} \sum_{\mathrm{i} \in \mathrm{V}} \mathrm{x}_{\mathrm{ijj}}=1 \quad \forall \mathrm{j} \in \mathrm{J} \\
& \sum_{\mathrm{m} \in \mathrm{M}} \mathrm{Zu}_{\mathrm{ijkm}} * \mathrm{mp}_{\mathrm{m}} \leq \mathrm{q}_{\mathrm{k}} * \mathrm{x}_{\mathrm{ijk}}, \forall \mathrm{i} \in \mathrm{V}, \forall \mathrm{j} \in \mathrm{V}, \forall \mathrm{k} \in \mathrm{K} \\
& \sum_{\mathrm{j} \in V} x_{i j k}=\sum_{j \in V} x_{j i k} \quad \forall \mathrm{i} \in \mathrm{V}, \forall \mathrm{k} \in \mathrm{K} \\
& \sum_{i \in \mathrm{I}} \sum_{j \in \mathrm{J}} x_{i j k} \leq 1 \forall \mathrm{k} \in \mathrm{K} \\
& \sum_{i \in S} \sum_{j \in S} x_{i j k} \leq|S|-1 \forall \mathrm{S} \subseteq \mathrm{J}, \forall \mathrm{k} \in \mathrm{K} \\
& \sum_{k \in K} \sum_{i \in V} z u_{i j k m}-\sum_{k \in K} \sum_{i \in V} z u_{j i k m}=d_{j m} \quad \forall \mathrm{j} \in \mathrm{J}, \forall \mathrm{m} \in \mathrm{M}
\end{aligned}
$$

$$
z u_{i j k m}=0 \quad \forall \mathrm{i} \in \mathrm{J}, \forall \mathrm{j} \in \mathrm{I}, \forall \mathrm{k} \in \mathrm{K}, \forall \mathrm{m} \in \mathrm{M}
$$

$$
\begin{aligned}
& z u_{i j k m} \leq m g * x_{i j k} \quad \forall \mathrm{i} \in \mathrm{V}, \forall \mathrm{j} \in \mathrm{V}, \forall \mathrm{k} \in \mathrm{K}, \forall \mathrm{m} \in \mathrm{M} \\
& z u_{i j k m} \geq 0 \quad \forall \mathrm{i} \in \mathrm{V}, \forall \mathrm{j} \in \mathrm{V}, \forall \mathrm{k} \in \mathrm{K}, \forall \mathrm{m} \in \mathrm{M} \quad(10)
\end{aligned}
$$

$$
x_{i j k} \in\{0,1\} \forall \mathrm{i} \in \mathrm{V}, \forall \mathrm{j} \in \mathrm{V}, \forall \mathrm{k} \in \mathrm{K}
$$


La función objetivo (1) minimiza la suma de dos costos distintos: el primero asociado con los arcos visitados por las rutas; y el segundo, con los costos fijos relacionados al uso de los vehículos como se definen en (Escobar, Linfati, Rodrigo, \& Toth, 2013). El grupo de restricciones (2) establece que cada cliente debe ser visitado estrictamente 1 vez por una ruta asignada a un solo vehículo $\mathrm{k} \in \mathrm{K}$. La restricción (3) se asocia con la capacidad de los vehículos. El grupo de restricciones (4) y (5) aseguran la continuidad de cada ruta y determina que cada una de ellas inicie y finalice en el depósito. La restricción de los subtours es satisfecha mediante el grupo de restricciones (6). El conjunto de restricciones (7) se asocian con la satisfacción de la demanda de cada producto por cada uno de los clientes. La restricción (8) evita el flujo de productos desde los clientes hacia el depósito. El grupo de restricciones (9) y (10) se asocian al acotamiento y no negatividad de las cantidades del producto $m$ a transportar de un nodo a otro en el vehículo k. Finalmente, el grupo de restricciones (11) hace referencia a la variable binaria del modelo para la asignación de rutas.

\section{Resultados y discusión}

Luego de haber definido los parámetros, variables, función objetivo y restricciones del modelo se procedió a codificarlo en GAMS y posteriormente ejecutado en un computador con procesador Intel Core i5 $(2.8 \mathrm{GHz})$ con 4 GB de memoria RAM, obteniendo una solución óptima. (Tablas 7, 8, 9, $10)$.

\section{Tabla 7}

Rutas establecidas y cantidades a enviar de cada producto.

\begin{tabular}{cccccccc}
\hline Ruta/producto & $\mathbf{1}$ & $\mathbf{2}$ & 3 & 4 & 5 & 6 & 7 \\
\hline$X_{134}$ & 810 & 746 & 0 & 0 & 320 & 275 & 300 \\
$X_{344}$ & 0 & 420 & 0 & 0 & 320 & 275 & 300 \\
$X_{414}$ & 0 & 0 & 0 & 0 & 0 & 0 & 0 \\
\hline
\end{tabular}

Nota. Fuente: construcción propia. 
Tabla 8

Rutas establecidas y cantidades a enviar de cada producto.

\begin{tabular}{cccccccc}
\hline Ruta/producto & $\mathbf{1}$ & $\mathbf{2}$ & $\mathbf{3}$ & $\mathbf{4}$ & $\mathbf{5}$ & $\mathbf{6}$ & $\mathbf{7}$ \\
\hline$X_{153}$ & 1550 & 245 & 480 & 240 & 0 & 0 & 0 \\
$X_{523}$ & 730 & 245 & 0 & 0 & 0 & 0 & 0 \\
$X_{213}$ & 0 & 0 & 0 & 0 & 0 & 0 & 0 \\
\hline
\end{tabular}

Nota. Fuente: construcción propia.

Tabla 9

Rutas establecidas y cantidades a enviar de cada producto.

\begin{tabular}{cccccccc}
\hline Ruta/producto & $\mathbf{1}$ & $\mathbf{2}$ & $\mathbf{3}$ & $\mathbf{4}$ & 5 & 6 & 7 \\
\hline$X_{162}$ & 0 & 350 & 200 & 100 & 0 & 0 & 350 \\
$X_{612}$ & 0 & 0 & 0 & 0 & 0 & 0 & 0 \\
\hline
\end{tabular}

Nota. Fuente: construcción propia.

Tabla 10

Rutas establecidas y cantidades a enviar de cada producto.

\begin{tabular}{cccccccc}
\hline Ruta/producto & $\mathbf{1}$ & $\mathbf{2}$ & $\mathbf{3}$ & $\mathbf{4}$ & $\mathbf{5}$ & $\mathbf{6}$ & $\mathbf{7}$ \\
\hline$X_{175}$ & 1650 & 250 & 150 & 70 & 0 & 0 & 150 \\
$X_{785}$ & 750 & 0 & 150 & 70 & 0 & 0 & 150 \\
$X_{815}$ & 0 & 0 & 0 & 0 & 0 & 0 & 0 \\
\hline
\end{tabular}

Nota. Fuente: construcción propia.

Esta solución establece 4 rutas para atender la demanda de los 7 clientes, como se evidencia en la figura 4. La primera es visitar a los clientes 3 y 4 en la ruta asignada al vehículo 4 . Como segunda ruta se establece atender a los clientes 5 y 2 en el vehículo 3. La tercera ruta visita al cliente 6 en el vehículo 2. Por último, la ruta 4 atiende a los clientes 7 y 8 en el vehículo 5. 


\section{Figura 4}

Cadena de suministro a nivel mundial y nacional.

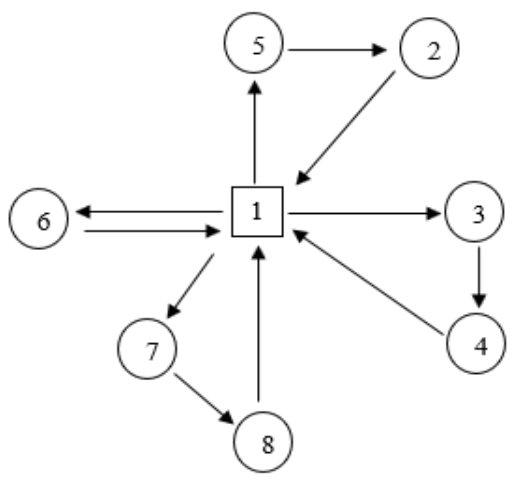

Nota. Fuente: construcción propia.

Este sistema de ruteo arroja un valor de $\mathrm{Z}$ de $\$ 3.135 .000$, los cuales corresponden a los costos logísticos de transporte.

Por tal razón, teniendo en cuenta la comparación de los costos actuales de transporte que la empresa presenta frente a los costos de transporte arrojados por el modelo, se puede decir que existe una diferencia de $\$ 415.000$ lo que equivale a una disminución del 11,69\% de ellos.

Costo de transporte actual: $\$ 3.550 .000$

Costo de transporte hallado: $\$ 3.135 .000$

\section{Conclusiones}

Tras la realización de este documento, a manera de conclusión, se puede decir que actualmente es necesario que tanto la alta gerencia como los empleados conozcan a fondo las actividades vinculadas a la cadena de suministro, debido a que esto le genera a la organización un valor agregado para la ventaja competitiva de la misma, teniendo en cuenta que la correcta gestión de la cadena de suministro es un factor clave para identificar, solucionar y prevenir problemáticas en los procesos vinculados a la operación de la empresa. Tener claro cómo opera en su totalidad cada tarea facilitará la toma de decisiones en cuanto a eficiencia, eficacia, sostenibilidad y sustentabilidad. 
Por otro lado, se puede decir que el aumento de la competitividad que se está viviendo actualmente en los mercados tanto nacionales como internacionales ha generado una gran inquietud en las organizaciones, haciéndolas caer en cuenta que, para perdurar y aumentar sus ganancias en ambientes con un nivel de exigencia mayor, es fundamental no quedarse con lo que pasa solo en las cuatro paredes de una planta. Al contrario, es de gran importancia el intercambiar ya sea información, recursos, entre otros elementos claves, con todo aquello que se encuentre vinculado a su cadena de suministro (proveedores, clientes, etc.), mediante la integración de sus actividades y la aplicación de nuevas técnicas innovadoras que aporten algún tipo de beneficio conjunto.

Ahora bien, de acuerdo a la problemática descrita, se recomienda a las organizaciones diseñar sistemas de ruteo estratégicos que permitan seleccionar la mejor ruta con el mínimo de costo, ya que este es uno de los problemas que mayormente se presentan en las empresas y, por ende, uno de los que mayor impacto genera.

Teniendo en cuenta lo anterior, se puede decir que el presente trabajo podrá servir como modelo o guía para el desarrollo de investigaciones enfocadas en esta misma área.

\section{Agradecimientos}

Para llevar a cabo el desarrollo de esta investigación fue necesaria la colaboración del ingeniero José Luis Ruiz Meza y la productora de ladrillos Cerámicas El Cinco, a los cuales se les agradece pues fueron quienes nos suministraron asesorías, información confidencial y el conocimiento indispensable para la elaboración de esta investigación.

\section{Referencias}

Ballou, R. (2004). Logística: Administración de la cadena de suministro. México: Prentice Hall.

Chopra, S., \& Meindl, P. (2016). Supply Chain Management: Strategy, Planning, and Operation (6ta ed.). México DF: Pearson educación.

Daza, J., Montoya, J., \& Narducci, F. (2013). Resolución del problema de enrutamiento de vehículos con limitaciones de capacidad utili- 
zando un procedimiento metaheurístico de dos fases. Revista EIA, 6(12), 23-38.

Escobar, J. W., Linfati, R., \& Toth, P. (2013). A two-phase hybrid heuristic algorithm for the capacitated location-routing problem. Computers E Operations Research, vol. 40, 70-79.

Gutiérrez, E., Fuquen, H., \& Abril , D. (2010). Planificación integrada de producción y distribución para un conglomerado industrial. Revista Facultad de Ingeniería Universidad de Antioquia(53), 88-105.

Joshi, S., \& Kaur, S. (2015). Comparative Analysis of Two Different Heuristics for Model of VRP. 2015 Second International Conference on Advances in Computing and Communication Engineering, 124-127. 